\title{
Should asymptomatic young men with iron deficiency anemia necessarily undergo endoscopy?
}

\author{
Nam Hee Kim ${ }^{1}$, Jung Ho Park ${ }^{2}$, Dong Il Park ${ }^{2}$, Chong Il Sohn ${ }^{2}$, Kyuyong Choi ${ }^{2}$, and Yoon Suk Jung ${ }^{2}$
}

${ }^{1}$ Preventive Healthcare Center, ${ }^{2}$ Division of Gastroenterology, Department of Internal Medicine, Kangbuk Samsung Hospital, Sungkyunkwan University School of Medicine, Seoul, Korea

Received: December 17, 2016 Revised : March 11, 2017 Accepted: April 3, 2017

\section{Correspondence to}

Yoon Suk Jung, M.D.

Division of Gastroenterology, Department of Internal Medicine, Kangbuk Samsung Hospital, Sungkyunkwan University School of Medicine, 29 Saemunan-ro, Jongno-gu, Seoul 03181, Korea Tel: +82-2-2001-8577

Fax: +82-2-2001-2049

E-mail:ys810.jung@samsung.com
Background/Aims: There has been no evidence for the necessity of endoscopy in asymptomatic young men with iron deficiency anemia (IDA). To determine whether endoscopy should be recommended in asymptomatic young men with IDA, we compared the prevalence of gastrointestinal (GI) lesions between young men (<50 years) with IDA and those without IDA.

Methods: We conducted a case-control study on asymptomatic young men aged < 50 years who underwent both esophagogastroduodenoscopy (EGD) and colonoscopy as part of a health checkup between 2010 and 2014.

Results: Of 77,864 participants, 128 (0.16\%) had IDA and 512 subjects without IDA were matched for several variables including age. Young men with IDA had a significantly higher proportion of colorectal cancer (CRC) (0.8\% vs. $0.0 \%, p=0.045)$, villous adenoma ( $0.8 \%$ vs. $0.0 \%, p=0.045$ ), and inflammatory bowel disease (IBD; $2.3 \%$ vs. $0.4 \%, p=0.025)$ than those without IDA. Additionally, the prevalence of advanced colorectal neoplasia (ACRN) tended to be higher in subjects with IDA than in those without IDA (3.1\% vs. $1.0 \%, p=0.084$ ). The prevalence of significant lower GI lesions including ACRN and IBD was higher in subjects with IDA than in those without IDA (5.5\% vs. 1.4\%, $p=0.011$ ). Regarding upper GI lesions, a positive association with IDA was observed only for gastric ulcer $(4.7 \%$ vs. $1.0 \%, p=$ 0.011).

Conclusions: GI lesions including CRC, villous adenoma, IBD, and gastric ulcer were more common in asymptomatic young men with IDA. Our results suggest that EGD and particularly colonoscopy should be recommended even in asymptomatic young men with IDA.

Keywords: Iron-deficiency anemia; Men; Duodenoscopy; Colonoscopy

\section{INTRODUCTION}

Iron deficiency anemia (IDA) occurs in $2 \%$ to $5 \%$ of adult men and postmenopausal women in the developed world $[1,2]$, and it is a common cause of referral to gastroenterologists [3]. While the most common cause of IDA in premenopausal women is menstrual blood loss, the most common cause in adult men and postmenopausal women is blood loss from the gastrointestinal (GI) tract [4-7]. IDA has been considered a red flag for the possible presence of serious GI disease, because a substantial proportion of asymptomatic gastric cancer, colorectal cancer (CRC), and precancerous lesions may present with IDA [8-10]. Therefore, it is essential to in- 
vestigate the origin of IDA through examination of both the upper and lower GI tract.

Accordingly, the current guidelines for the management of IDA recommend, based on the results of randomized controlled trials or cohort studies, that upper and lower GI tract examinations should be considered in all male and all postmenopausal female patients in whom IDA has been confirmed, unless there is a history of significant overt non-GI blood loss [7]. The current guidelines recommend that young men with IDA should be investigated in the same manner as older men with IDA, although there are no data on the yield of investigation in young men with IDA [7]. However, the incidence of significant GI pathology such as gastric cancer and CRC in younger individuals is much lower than that in older individuals [11,12]. It appears to be reasonable to recommend GI investigations in all postmenopausal female patients with IDA because the average age of menopause is around 50 years, whereas the current guidelines that recommend GI investigation of IDA for all men regardless of age may be unreasonable. The yield of upper and lower GI investigations in young men with IDA may be lower than that in older men with IDA. However, evidence for the necessity of endoscopy in young men with IDA is significantly lacking, which led us to question whether it is reasonable to recommend esophagogastroduodenoscopy (EGD) and colonoscopy for all young men with IDA.

Therefore, the aim of the present study was to evaluate whether young men with IDA indeed have a greater number of significant upper or lower GI lesions compared to those without IDA, and to determine whether young men with IDA should undergo both EGD and colonoscopy. To obtain more reliable answers to our questions, we investigated asymptomatic young men aged $<50$ years who underwent both EGD and colonoscopy.

\section{METHODS}

\section{Study population}

We retrospectively analyzed data obtained from a prospectively established cohort. The present study population consisted of asymptomatic male participants aged $<50$ years who had undergone both EGD and colonos- copy as part of a comprehensive health screening program at Kangbuk Samsung Hospital, Seoul and Suwon, Korea, from 2010 to 2014 ( $n=86,153$ ). The purpose of the screening program was to promote health through a regular health checkup and to enhance early detection of existing diseases. In Korea, the Industrial Safety and Health Law requires employees to participate in annual or biennial health examinations. About $80 \%$ of participants were employees of various companies and local governmental organizations and their spouses, with the remaining participants registering individually for the program. Before the performance of endoscopy, interviews by general practitioners were conducted to ensure that all participants were asymptomatic (i.e., no abdominal pain, melena, or hematochezia). Individuals with overt GI symptoms were urged to seek medical care.

For this analysis, the exclusion criteria were as follows: a history of CRC or colorectal surgery $(n=171)$, a history of stomach cancer or gastric surgery $(n=189)$, diagnosis of renal failure (estimated creatinine clearance rate $<30$ using the Cockcroft-Gault formula) during this study (n $=6$ ), poor bowel preparation $(n=7,651)$, lack of an adequate biopsy $(\mathrm{n}=141)$, and missing data on IDA $(\mathrm{n}=$ 131). Ultimately, the total number of eligible subjects for the study was 77,864. Among these, 128 (0.16\%) had IDA. In order to control the potential factors associated with

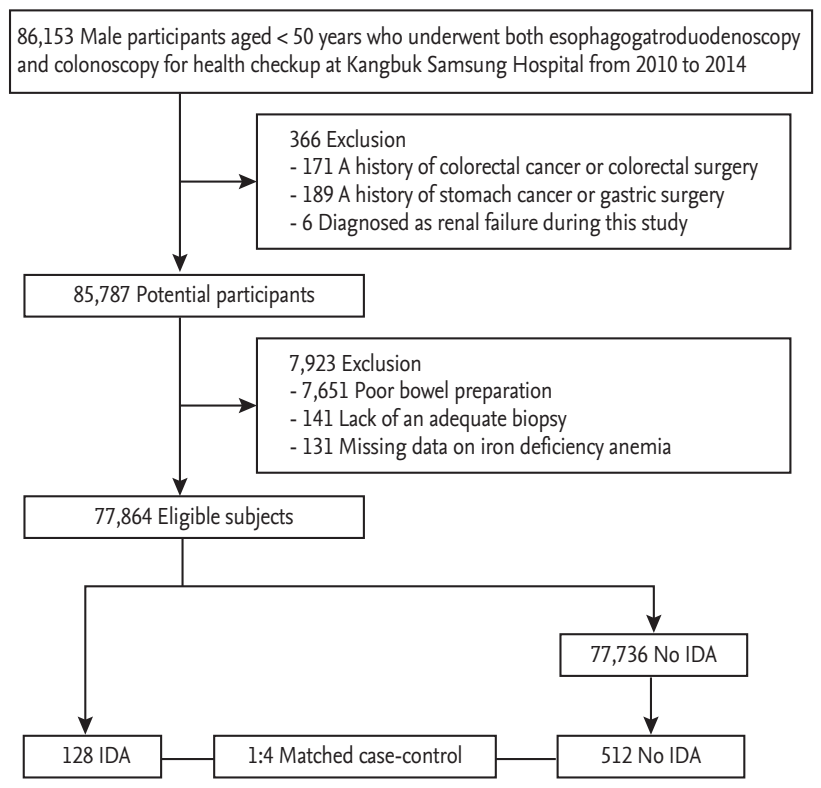

Figure 1. Flow diagram illustrating the selection of study subjects. IDA, iron deficiency anemia. 
the risk of colorectal neoplasia (CRN), four controls for each case patients who were matched for age, smoking status, family history of CRC, and use of nonsteroidal anti-inflammatory drugs (NSAIDs) were selected from the subjects without IDA $(\mathrm{n}=512)$ (Fig. 1 ).

This study was approved by the Institutional Review Board of Kangbuk Samsung Hospital (IRB file number: 2016-04-038, approved on 2016-04-25). The requirement for informed consent was waived because only de-identified data were retrospectively accessed.

\section{Measurements and definitions}

Data on medical history and health-related behaviors were collected through a standardized, self-administered questionnaire, whereas physical measurements such as height and weight were assessed by a trained staff member. Participants' smoking status was categorized into never, formerly, or currently. Family history of CRC or gastric cancer was defined as CRC or gastric cancer in one or more first-degree relatives at any age. Self-reported use of NSAIDs or antiplatelet or anticoagulant medications (regular use over the previous month) was also assessed.

Hypertension was defined as systolic blood pressure $\geq 140 \mathrm{mmHg}$, diastolic blood pressure $\geq 90 \mathrm{mmHg}$, or current use of antihypertensive medication. Diabetes mellitus was defined as a fasting blood glucose $\geq 126$ $\mathrm{mg} / \mathrm{dL}$, hemoglobin A1c $\geq 6.5 \%$, or current use of insulin or antidiabetic mediations. Obesity was defined as body mass index $(\mathrm{BMI}) \geq 25 \mathrm{~kg} / \mathrm{m}^{2}$, which is the proposed cutoff for the diagnosis of obesity in Asians [13]. BMI was calculated by dividing the measured weight $(\mathrm{kg})$ by the square of the height $\left(\mathrm{m}^{2}\right)$.

Anemia was defined as hemoglobin less than $13 \mathrm{~g} / \mathrm{dL}$, and IDA was defined as anemia accompanied by at least one of the following criteria characterizing iron deficiency: (1) serum ferritin < $15 \mathrm{ng} / \mathrm{mL}$; and (2) transferrin saturation (serum iron/total iron binding capacity) < $16 \%[2,14]$.

\section{Endoscopy and histologic examination}

All EGD and colonoscopies were performed by experienced board-certified endoscopists, using a GIF-H260 esophagoduodenoscope and EVIS Lucera CV-26o colonoscope (Olympus Medical Systems, Tokyo, Japan), respectively. All participants took $4 \mathrm{~L}$ of polyethylene gly- col solution for bowel preparation. EGD was performed first, immediately followed by colonoscopy in all cases.

The following lesions were considered as upper GI causes of IDA on EGD: erosion, ulcers, adenoma, cancer, polyps, and angiodysplasia. On colonoscopy, the following lesions were considered as lower GI sources of IDA: adenoma, CRC, and inflammatory bowel disease (IBD).

All endoscopically abnormal lesions were biopsied or removed. All specimens obtained from biopsy, polypectomy, or endoscopic mucosal resections were evaluated by experienced GI pathologists via histopathological examination.

In the case of gastric ulcer, to differentiate benign or malignant gastric ulcers, both endoscopic and histopathologic examinations were assessed. Only benign gastric ulcer was defined as gastric ulcer, while malignant gastric ulcer was defined as gastric cancer.

Advanced adenoma was defined as the presence of one of the following features: $>10 \mathrm{~mm}$ diameter, tubulovillous or villous structure, and high-grade dysplasia (HGD) [15]. High-risk adenoma was defined as advanced adenoma or 3 or more adenomas [15]. CRN was defined as a cancer or any adenoma, and advanced colorectal neoplasia (ACRN) was defined as a cancer or advanced adenoma [16]. Significant lower GI findings were regarded as ACRN and IBD.

\section{Statistical analysis}

Data are expressed as mean \pm standard deviation or frequency (\%). Baseline characteristics and GI lesions were compared between subjects with versus without IDA by chi-squared analysis or Fisher exact test for categorical variables and by Student $t$ test for continuous variables. All reported $p$ values are two-tailed, and $p$ values $<0.05$ were considered statistically significant. SPSS version 18.o (SPSS Inc., Chicago, IL, USA) was used for statistical analyses.

\section{RESULTS}

\section{Baseline characteristics of the study population}

Of 77,864 participants, 128 (0.16\%) had IDA. The baseline characteristics of 128 subjects with IDA were compared with 512 subjects without IDA who were matched for age, smoking status, family history of CRC, and use 
of NSAIDs (Table 1). The mean age of the study participants was 39.4 years, and the proportions of subjects aged 20 to 29 years, 30 to 39 years, and 40 to 49 years were $3.1 \%, 46.1 \%$, and 50.8\%, respectively. There were no significant differences in other baseline characteristics including family history of gastric cancer, use of antiplatelet or anticoagulant medications, obesity, fatty liver, hypertension, and diabetes mellitus between subjects with IDA and those without IDA.

Table 1. Baseline characteristics of the study population according to presence of iron deficiency anemia

\begin{tabular}{|c|c|c|c|}
\hline Variable & $\operatorname{IDA}(\mathrm{n}=128)$ & No IDA $(n=512)$ & $p$ value \\
\hline Age, yr & $39.4 \pm 5.9$ & $39.4 \pm 5.9$ & 1.000 \\
\hline $20-29$ & $4(3.1)$ & $16(3.1)$ & 1.000 \\
\hline $30-39$ & $59(46.1)$ & $235(46.1)$ & \\
\hline $40-49$ & $65(50.8)$ & $260(50.8)$ & \\
\hline Current or ex-smoker & $77 / 126(61.1)$ & $308 / 504(61.1)$ & 1.000 \\
\hline Family history of CRC & $5(3.9)$ & $20(3.9)$ & 1.000 \\
\hline Family history of gastric cancer & $14(10.9)$ & $29(5 \cdot 7)$ & 0.053 \\
\hline Use of NSAIDs & $3(2.3)$ & $12(2.3)$ & 1.000 \\
\hline Use of antiplatelet or anticoagulant & $4(3.1)$ & $17(3 \cdot 3)$ & 1.000 \\
\hline Body mass index, $\mathrm{kg} / \mathrm{m}^{2}$ & $24.7 \pm 3.2$ & $24.6 \pm 2.9$ & 0.775 \\
\hline Obesity, $\geq 25 \mathrm{~kg} / \mathrm{m}^{2}$ & $57(44.9)$ & $210(41.0)$ & 0.482 \\
\hline Fatty liver & $50(39.1)$ & $218(42.6)$ & 0.471 \\
\hline Hypertension & $24(18.8)$ & $71(13.9)$ & 0.165 \\
\hline Diabetes mellitus & $4(3.1)$ & $24(4.7)$ & 0.440 \\
\hline
\end{tabular}

Values are presented as mean \pm SD or number (\%). Matching variables in case-control were age, smoking status, family history of CRC, and use of NSAIDs.

IDA, iron deficiency anemia; CRC, colorectal cancer; NSAID, nonsteroidal anti-inflammatory drug.

Table 2. Upper gastrointestinal lesions according to presence of iron deficiency anemia

\begin{tabular}{|c|c|c|c|}
\hline Variable & $\operatorname{IDA}(\mathrm{n}=128)$ & No IDA $(n=512)$ & $p$ value \\
\hline Gastric cancer & o & $1(0.2)$ & 1.000 \\
\hline Gastric adenoma & o & o & NA \\
\hline Gastric polyp & $3(2.3)$ & $10(2.0)$ & 0.730 \\
\hline Gastric ulcer & $6(4.7)$ & $5(1.0)$ & 0.011 \\
\hline Gastric erosion & $31(24.2)$ & $103(20.1)$ & 0.308 \\
\hline Duodenal adenoma & o & o & NA \\
\hline Duodenal polyp & o & $4(0.8)$ & 0.589 \\
\hline Duodenal ulcer & $1(0.8)$ & $4(0.8)$ & 1.000 \\
\hline Duodenal erosion & o & $2(0.4)$ & 1.000 \\
\hline Esophageal ulcer & 0 & $1(0.2)$ & 1.000 \\
\hline Angiodysplasia & $1(0.8)$ & 0 & 0.200 \\
\hline Upper gastrointestinal lesions, total & $38(29.7)$ & $121(23.6)$ & 0.156 \\
\hline
\end{tabular}

Values are presented as number (\%). Matching variables in case-control were age, smoking status, family history of colorectal cancer, and use of nonsteroidal anti-inflammatory drugs.

IDA, iron deficiency anemia; NA, not available. 
Table 3. Lower gastrointestinal lesions according to presence of iron deficiency anemia

\begin{tabular}{|c|c|c|c|}
\hline Variable & $\operatorname{IDA}(n=128)$ & No IDA $(n=512)$ & $p$ value \\
\hline Advanced CRN & $4(3.1)$ & $5(1.0)$ & 0.084 \\
\hline Advanced adenoma & $3(2.3)$ & $5(1.0)$ & 0.202 \\
\hline High-grade dysplasia & 0 & 0 & NA \\
\hline TVA or VA & $1(0.8)$ & 0 & 0.045 \\
\hline Size $\geq 10 \mathrm{~mm}$ & $3(2.3)$ & $5(1.0)$ & 0.202 \\
\hline 3 or more adenomas & $1(0.8)$ & $4(0.8)$ & 1.000 \\
\hline High-risk adenoma & $3(2.3)$ & $9(1.8)$ & 0.714 \\
\hline Cancer & $1(0.8)$ & 0 & 0.045 \\
\hline Non-advanced adenoma & $12(9.4)$ & $62(12.1)$ & 0.387 \\
\hline Overall CRN & $16(12.5)$ & $67(13.1)$ & 0.860 \\
\hline Inflammatory bowel disease & $3(2.3)$ & $2(0.4)$ & 0.025 \\
\hline Ulcerative colitis & $1(0.8)$ & $2(0.4)$ & 0.489 \\
\hline Crohn's disease & $2(1.6)$ & 0 & 0.005 \\
\hline Lower GI lesions, total & $18(14.1)$ & $69(13.5)$ & 0.863 \\
\hline Significant lower GI lesions & $7(5 \cdot 5)$ & $7(1.4)$ & 0.011 \\
\hline
\end{tabular}

Values are presented as number (\%). Significant lower GI findings included advanced CRN and inflammatory bowel disease. Matching variables in case-control were age, smoking status, family history of colorectal cancer, and use of nonsteroidal anti-inflammatory drugs.

IDA, iron deficiency anemia; CRN, colorectal neoplasia; NA, not available; TVA, tubulovillous adenoma; VA, villous adenoma; GI, gastrointestinal.

\section{Upper GI lesions and IDA}

Upper GI lesions observed on EGD according to the presence of IDA are presented in Table 2. Subjects with IDA had a significantly higher proportion of gastric ulcer compared to those without IDA ( $4.7 \%$ vs. $1.0 \%$, p $=0.011)$. However, the prevalence of other upper GI lesions including gastric cancer, gastric adenoma, gastric polyp, gastric erosion, duodenal adenoma, duodenal polyp, duodenal ulcer, duodenal erosion, esophageal ulcer, and angiodysplasia was not significantly different between subjects with IDA and those without IDA. The proportion of total upper GI lesions was also not different between subjects with IDA and those without IDA (29.7\% vs. $23.6 \%, p=0.156$ ).

\section{Lower GI lesions and IDA}

Colonoscopic and histologic findings according to the presence of IDA are presented in Table 3. The prevalence of adenoma with villous component (o.8\% vs. $0 \%$, $p=0.045)$, cancer ( $0.8 \%$ vs. $0 \%, p=0.045)$, and IBD $(2.3 \%$ vs. $0.4 \%, p=0.025)$ was significantly higher in subjects with IDA compared to those without IDA. In IBD, the proportion of Crohn's disease ( $1.6 \%$ vs. $0.0 \%, p=0.005$ ) was significantly higher in subjects with IDA compared to those without IDA, whereas the proportion of ulcerative colitis ( $0.8 \%$ vs. $0.4 \%, p=0.489$ ) did not significantly differ between subjects with IDA and those without IDA. The prevalence of ACRN tended to be higher in subjects with IDA than in those without IDA although the difference did not reach statistical significance (3.1 $\%$ vs. $1.0 \%, p=0.084$ ). However, the prevalence of adenoma with HGD, adenoma with > $10 \mathrm{~mm}$ diameter, 3 or more adenomas, and non-advanced adenoma did not significantly differ between subjects with IDA and those without IDA. The proportion of total lower GI lesions was not different between subjects with IDA and those without IDA (14.1\% vs. $13.5 \%, p=0.863$ ), whereas the proportion of significant lower GI lesions including ACRN and IBD was higher in subjects with IDA than in those without IDA ( $5.5 \%$ vs. $1.4 \%, p=0.011$ ).

\section{DISCUSSION}

This study including asymptomatic screened men aged $<50$ years evaluated whether young men with IDA have 
a greater number of significant upper and lower GI lesions compared to those without IDA. To the best of our knowledge, this is first study to focus on comparison of the prevalence of GI lesions between subjects with and without IDA among a population of young men and to evaluate the necessity of endoscopy in young men. We found that young men with IDA had a significantly higher proportion of CRC, villous adenoma, and IBD than did those without IDA. Additionally, the prevalence of significant lower GI lesions including ACRN and IBD was higher in subjects with IDA than in those without IDA. However, a positive association between IDA and upper GI lesions was observed only in the case of gastric ulcer. Our findings suggest that EGD, and especially colonoscopy, may be helpful to identify clinically significant sources of IDA even in asymptomatic young men aged $<50$ years.

IDA is one of the common medical conditions encountered in clinical practice and accounts for approximately one half of cases of anemia [17,18]. IDA is not a disease in itself, but may be a manifestation of a serious underlying disease such as malignancy, and searching for causes of IDA is therefore crucial. The etiologies of IDA differ with age and gender, and chronic occult GI bleeding is the most common cause of IDA in adult men and postmenopausal women. Many studies have reported an association between IDA and GI malignancy. One prospective study including 100 subjects with IDA showed that significant GI lesions including cancer were frequently found in patients with IDA (62\%) [19]. Another prospective study in which GI investigations were performed in 695 patients with IDA showed that GI cancer was diagnosed with significant frequency (11.2\%, $78 / 695)$ in patients with IDA [20]. Additionally, a population-based study including 9,024 subjects showed that among men and postmenopausal women, GI malignancies are significantly more common in those with IDA than in those without IDA, and subjects with IDA are at increased risk of diagnosis of GI cancer within the subsequent 2 years [21]. Based on these previous studies, the possible existence of a malignancy as the source of IDA is of great concern and accordingly, current guidelines recommend that upper and lower GI investigation should be considered in all postmenopausal females and all male patients with IDA, unless there is evidence of significant overt non-GI blood loss [7]. However, in previous studies, the majority of study participants were over 50 years of age.

There have been no evidence-based recommendations focusing on young men ( $<50$ years) with IDA due to the paucity of reliable clinical data in this population. Current guidelines recommend that young men with IDA should be investigated in the same manner as older men with IDA, although there are few data to support this approach (recommendation with Level of Evidence C) $[7,22]$. To date, only a few studies have evaluated the prevalence of GI lesions among young men ( $<50$ years) with IDA. One Israeli study including 347 young men (18 to 40 years) with IDA demonstrated that significant GI lesions were diagnosed in a large portion of the study population $(62 \%, 217 / 347)[23]$. However, the study emphasized GI symptoms as a predictor of significant GI lesions, and suggested that GI evaluation should be performed in symptomatic young men [23]. In other words, the study did not provide an obvious answer to the question of the preferred investigation strategy in asymptomatic young men with IDA. A British study reported that significant GI findings were frequent in young men (< 50 years) with IDA $(20.6 \%, 6 / 29)$ and suggested that endoscopy is an appropriate strategy of investigation for men $<50$ years of age with IDA [24]. However, these two studies did not compare the prevalence of GI lesions between subjects with versus without IDA and reported only the proportion of GI lesions in young men with IDA. Moreover, the sample sizes of the studies were quite small. Therefore, it is difficult to draw definitive conclusions from these two studies regarding whether endoscopy should be recommended in asymptomatic young men with IDA.

One of the strengths of our study is that the sample size is not small. Of 77,864 participants, 128 with IDA were observed and these patients were compared with 512 subjects without IDA who were matched for several variables including age. Therefore, we think that our study provides more reliable information on the yield of GI investigations among young men with IDA than previous studies. Furthermore, this is first study to demonstrate that significant lower GI lesions including CRC, villous adenoma, and IBD were more common in subjects with IDA compared to those without IDA, even in young men without symptoms. In addition, gastric ulcer, which requires treatment, was more frequent in 
asymptomatic young men with IDA compared to those without IDA.

As the incidence of GI malignancies such as gastric cancer and CRC increases progressively with age, the likelihood of developing GI malignancy is relatively much lower in young men $<50$ years than in men > 50 years $[11,12]$. Therefore, physicians may agonize over whether both EGD and colonoscopy should necessarily be performed in the case of young men with IDA. However, the present study showed that only $0.16 \%$ of men $<50$ years of age had IDA. Because only a very small proportion of men $<50$ years of age had IDA, it may be reasonable and cost-effective to recommend EGD and colonoscopy for all young men with IDA. Based on our findings that young men with IDA had higher rates of CRC and villous adenoma than did young men without IDA, while the prevalence of gastric cancer did not differ between the two groups, colonoscopy rather than EGD may be a more important examination for young men with IDA.

In the present study, the prevalence of villous adenoma was significantly higher in patients with IDA compared to those without IDA. In addition, the prevalence of advanced adenoma, i.e., adenoma with $>10 \mathrm{~mm}$ diameter was slightly higher in patients with IDA than in those without IDA (2.3\% vs. $1.0 \%)$ although it was statistically insignificant. However, the prevalence of non-advanced adenoma was not higher in patients with IDA compared to those without IDA (9.4\% vs. 12.1\%). Although it is difficult to draw definitive conclusion from our results, advanced adenoma may affect the expression of IDA in asymptomatic patients. Adenoma with more advanced histology and/or larger size may be expected to have increased vascularity and thus is more likely to result in bleeding. Advanced adenoma may cause a slow, insidious chronic blood loss, so patients may not be aware of the lower GI bleeding symptoms such as hematochezia.

Another finding in the present study is that the prevalence of IBD, especially Crohn's disease was significantly higher in subjects with IDA compared to those without IDA. However, the proportion of ulcerative colitis did not differ between subjects with IDA and those without IDA. It seems to reflect the fact that IDA can be the important early manifestation of Crohn's disease [25,26].

There are several limitations in the present study. First, the study was hospital-based rather than popula- tion-based, and our cohort was recruited at two medical centers in Korea. These factors may result in some degree of selection bias. Readers should thus take care not to generalize on the basis of our results alone. Second, we did not evaluate non-bleeding-associated diseases such as atrophic gastritis and Helicobacter pylori gastritis that could present as IDA through malabsorption of iron [27,28]. Finally, we did not perform small bowel evaluation including routine duodenal biopsy for screening for celiac disease, even though current guidelines recommend that all patients with IDA should be screened for celiac disease [7]. However, in contrast to Western populations, celiac disease is extremely rare in Asian populations [29]. Moreover, the reported yield of small bowel investigation in obscure GI bleeding is quite low [30]. Therefore, the incidence of small bowel lesions as a source of IDA might be negligible.

In conclusion, young men with IDA had a significantly higher proportion of CRC, villous adenoma, IBD, and gastric ulcer compared to those without IDA. Our results suggest that EGD and especially colonoscopy should be recommended for the detection of significant GI lesions even in asymptomatic young men with IDA.

\section{KEY MESSAGE}

1. Young men with iron deficiency anemia (IDA) had a significantly higher proportion of colorectal cancer, villous adenoma, and inflammatory bowel disease (IBD) than did those without IDA.

2. The prevalence of significant lower gastrointestinal (GI) lesions including advanced colorectal neoplasia and IBD was higher in subjects with IDA than in those without IDA.

3. A positive association between IDA and upper GI lesions was observed only in the case of gastric ulcer.

4. Esophagogastroduodenoscopy and particularly colonoscopy should be recommended even in asymptomatic young men with IDA.

\section{Conflict of interest}

No potential conflict of interest relevant to this article was reported. 


\section{REFERENCES}

1. Guralnik JM, Eisenstaedt RS, Ferrucci L, Klein HG, Woodman RC. Prevalence of anemia in persons 65 years and older in the United States: evidence for a high rate of unexplained anemia. Blood 2004;104:2263-2268.

2. World Health Organization. Iron Deficiency Anaemia Assessment, Prevention and Control: A Guide for Programme Managers. Geneva: World Health Organization, 2001.

3. McIntyre AS, Long RG. Prospective survey of investigations in outpatients referred with iron deficiency anaemia. Gut 1993;34:1102-1107.

4. Looker AC, Dallman PR, Carroll MD, Gunter EW, Johnson CL. Prevalence of iron deficiency in the United States. JAMA 1997;277:973-976.

5. Andrews NC. Disorders of iron metabolism. N Engl J Med 1999;341:1986-1995.

6. Rockey DC. Occult gastrointestinal bleeding. N Engl J Med 1999;341:38-46.

7. Goddard AF, James MW, McIntyre AS, Scott BB; British Society of Gastroenterology. Guidelines for the management of iron deficiency anaemia. Gut 2011;60:1309-1316.

8. Lindsay JO, Robinson SD, Jackson JE, Walters JR. The investigation of iron deficiency anemia: a hospital based audit. Hepatogastroenterology 1999;46:2887-2890.

9. Harris GJ, Simson JN. Causes of late diagnosis in cases of colorectal cancer seen in a district general hospital over a 2-year period. Ann R Coll Surg Engl 1998;80:246-248.

10. Goodman D, Irvin TT. Delay in the diagnosis and prognosis of carcinoma of the right colon. Br J Surg 1993;80:1327-1329.

11. Inra JA, Syngal S. Colorectal cancer in young adults. Dig Dis Sci 2015;60:722-733.

12. Karimi P, Islami F, Anandasabapathy S, Freedman ND, Kamangar F. Gastric cancer: descriptive epidemiology, risk factors, screening, and prevention. Cancer Epidemiol Biomarkers Prev 2014;23:700-713.

13. Wen CP, David Cheng TY, et al. Are Asians at greater mortality risks for being overweight than Caucasians? Redefining obesity for Asians. Public Health Nutr 2009;12:497506.

14. Camaschella C. Iron-deficiency anemia. N Engl J Med 2015;372:1832-1843.

15. Lieberman DA, Rex DK, Winawer SJ, et al. Guidelines for colonoscopy surveillance after screening and pol- ypectomy: a consensus update by the US Multi-Society Task Force on Colorectal Cancer. Gastroenterology 2012;143:844-857.

16. Kwah J, Schroy PC 3rd, Jacobson BC, Calderwood AH. Whites and blacks have similar risk of metachronous advanced colorectal neoplasia. Dig Dis Sci 2014;59:2264-2271.

17. Short MW, Domagalski JE. Iron deficiency anemia: evaluation and management. Am Fam Physician 2013;87:98104.

18. Johnson-Wimbley TD, Graham DY. Diagnosis and management of iron deficiency anemia in the 21st century. Therap Adv Gastroenterol 2011;4:177-184.

19. Rockey DC, Cello JP. Evaluation of the gastrointestinal tract in patients with iron-deficiency anemia. N Engl J Med 1993;329:1691-1695.

20. James MW, Chen CM, Goddard WP, Scott BB, Goddard AF. Risk factors for gastrointestinal malignancy in patients with iron-deficiency anaemia. Eur J Gastroenterol Hepatol 2005;17:1197-1203.

21. Ioannou GN, Rockey DC, Bryson CL, Weiss NS. Iron deficiency and gastrointestinal malignancy: a population-based cohort study. Am J Med 2002;113:276-280.

22. Peytremann-Bridevaux I, Arditi C, Froehlich F, et al. Appropriateness of colonoscopy in Europe (EPAGE II): iron-deficiency anemia and hematochezia. Endoscopy 2009;41:227-233.

23. Carter D, Levi G, Tzur D, Novis B, Avidan B. Prevalence and predictive factors for gastrointestinal pathology in young men evaluated for iron deficiency anemia. Dig Dis Sci 2013;58:1299-1305.

24. Landy J, Macfarlane B. Synchronous bidirectional endoscopy for iron deficiency anaemia: is it appropriate for patients under 50? Postgrad Med J 2010;86:338-340.

25. Lucendo AJ, Arias A, Roncero O, et al. Anemia at the time of diagnosis of inflammatory bowel disease: prevalence and associated factors in adolescent and adult patients. Dig Liver Dis 2017;49:405-411.

26. Lee DS, Bang KB, Kim JY, et al. The prevalence and clinical characteristics of anemia in Korean patients with inflammatory bowel disease. Intest Res 2016;14:43-49.

27. Marignani M, Delle Fave G, Mecarocci S, et al. High prevalence of atrophic body gastritis in patients with unexplained microcytic and macrocytic anemia: a prospective screening study. Am J Gastroenterol 1999;94:766-772.

28. Choe YH, Kim SK, Son BK, Lee DH, Hong YC, Pai SH. Randomized placebo-controlled trial of Helicobacter 
pylori eradication for iron-deficiency anemia in preadolescent children and adolescents. Helicobacter 1999;4:135139 .

29. Gujral N, Freeman HJ, Thomson AB. Celiac disease: prevalence, diagnosis, pathogenesis and treatment. World J
Gastroenterol 2012;18:6036-6059.

30. Fireman Z, Kopelman Y. The role of video capsule endoscopy in the evaluation of iron deficiency anaemia. Dig Liver Dis 2004;36:97-102. 